\title{
Numerical considerations in spectral multi-domain methods for BiGlobal instability analysis of open cavity configurations
}

\author{
J. de Vicente, E. Valero and V. Theofilis
}

School of Aeronautics, Universidad Politécnica de Madrid

javier@dmae.upm.es

Summary. A novel approach for the solution of the viscous incompresible and/or compressible BiGlobal eigenvalue problems (EVP) in complex open cavity domains is discussed. The algorithm is based on spectral multidomain spatial discretization, decomposing space into rectangular subdomains which are resolved by spectral collocation based on Chebyshev polynomials. The eigenvalue problem is solved by Krylov subspace iteration. Here particular emphasis is placed on aspects of the parallel developments that have been necessary, on account of the high computing demands placed on the solver, as ever more complex "T-store" configurations are addressed.

\section{Theory}

\section{Spectral collocation approximation}

A Chebyshev spectral expansion of the function $u(x)$ is considered on the Gauss-Lobatto nodes,

$$
u_{N}(x)=\sum_{j=0}^{N} h_{j}(x) u\left(x_{j}\right)
$$

being $h_{j}(x)$ the Lagrange interpolation functions. The unknowns become the values of $u(x)$ at the grid points. Differentiation is introduced by using the interpolation polynomial which permits expressing derivatives as

$$
U=\mathcal{D} U, \quad U^{(p)}=\mathcal{D}^{(p)} U
$$

Using this technique, the solution of an eigenvalue problem is reduced to constructing the matrix $\mathcal{L}$ of the differential operator and manipulate it properly in order to impose boundary conditions. The extension of this one-dimensional idea to several Cartesian spatial dimensions is straightforward. 


\section{BiGlobal Theory}

Linear stability analysis in the BiGlobal framework involves the substitution of a decomposition of any of the independent flow variables into the equations of motion [1]. All quantities are considered to be composed of an $O(1)$ steady twodimensional basic state and $O(\epsilon)$ unsteady three-dimensional perturbations, according to the BiGlobal Ansatz

$$
\mathbf{q}(x, y, z, t)=\overline{\mathbf{q}}(x, y)+\epsilon \hat{\mathbf{q}}(x, y) \exp i(\beta z-\Omega t)
$$

for the determination of the complex eigenvalue $\Omega . \Omega_{r} \equiv \Re\{\Omega\}$ represents a frequency and $\Omega_{i} \equiv \Im\{\Omega\}$ is the amplification/damping rate of the disturbance, while barred and hatted quantities denote basic and disturbance flow, respectively. Discretization of the linearized equations of motion leads to the two-dimensional BiGlobal eigenvalue problem (EVP)

$$
A x=\Omega M x .
$$

\section{Spectral multi-domain discretization}

Multidomain spatial discretization divides the space into rectangular subdomains each resolved by spectral collocation. Once the two-dimensional BiGlobal eigenvalue problem has been formed for each domain boundary and interface conditions are imposed in order to form the global matrix discretizing the eigenvalue problem. There are several choices in the eigenvalue recovering algorithm. The storage of the matrix elements, and the use of different parallel machines determines the final algorithm, as schematically shown in figure 1 .

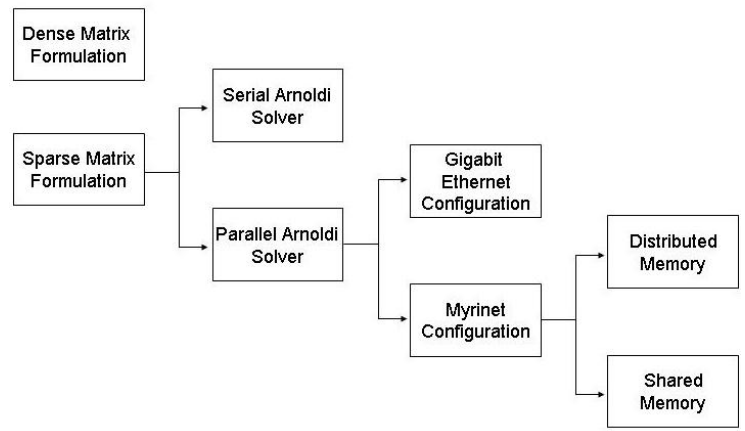

Fig. 1. Computational choices for the solution of the BiGlobal EVP 


\section{Parallel approach}

Once the matrix discretizing the EVP is stored in the CCF, it is solved by Krilov subspace iteration in two stages. First the matrix is LU-decomposed using SuperLU [2], which is a general purpose library for the direct solution of large, sparse, nonsymmetric systems of linear equations on high performance parallel machines. The library routines also perform an LU decomposition with partial pivoting and triangular system solves through forward and back substitution. This LU-decomposition is fed into an Arnoldi iteration to recover the leading eigenvalues. However, storing the LU-decomposition is itself demanding in memory; that associated with the T-store configuration shown in figure 2 requires an additional 2 Gbytes. Consequently, in all but the lowest Reynolds numbers ${ }^{1}$, the LU decomposition must be performed in parallel.

\footnotetext{
Gigabit Cluster

12 Intel Xeon compute nodes, each featuring:

- 2 single-core 32-bit Pentium

- 2GB DDR memory

- Gigabit Ethernet

Myrinet Cluster

256 IBM BladeCenter JS20 compute nodes, each featuring:

- 2 single-core, 2.20Ghz 64-bit PowerPC 970FX - 4GB PC2700 ECC DDR memory

- Dual Gigabit Ethernet with Myrinet interface
}

Shared-memory cluster

2 HP Integrity Superdome compute nodes, each featuring:

- 64 Itanium2, $1.5 \mathrm{Ghz}$

- 384GB DDR memory

Table 1. Computing cluster characteristics: Gigabit and Myrinet clusters feature distributed-memory.

\section{Hardware considerations}

Parallel performance broads in one side the scope of tackled problems, involving however some new difficulties: new programing strategy compatible with MPI structure and also a greater dependency on hardware in the sense of not only processors features but net architecture and comunications among processors. Example results for the solution of the (large) linear system $A x=b$ have been obtained on two different clusters, one denoted "Gigabit Ethernet", and one denoted "Myrinet". The characteristics of these distributed-memory machines are summarized in table 1 ; results obtained, shown in table 2, show the dependence of the performance of the SuperLU algorithm on the architecture chosen. Analogous results have been obtained on the Myrinet cluster, using one of the matrices from the validation suite of the CCF format. Results

\footnotetext{
${ }^{1}$ such cases have been presented elsewhere[3]
} 
shown in table 3 show that the theoretical linear speed-up is achieved, as long as sections of the matrix are kept in the (shared) memory of each computing node. This tendency stops as long as the number of processors increases beyond two and communication amongst nodes becomes the determining factor; figure 3 shows this result in graphical form.

These results for the solution of the linear system have led to the conclusion that use of sparse linear solvers on distributed-memory machines may not be as competitive as their counterparts on shared-memory architectures. Efforts are currently underway to develop a new version of the EVP solver for the BiGlobal instability problem in shared-memory parallel machines, such as the one indicated in table 1.

Table 2. Performance of the parallel LU-decomposition $A x=b$, in which $A$ features $O\left(10^{10}\right)$ non-zero elements

\begin{tabular}{|c|c|c|c|c|c|}
\hline & & \multicolumn{2}{|c|}{ Gigabit Cluster } & \multicolumn{2}{|c|}{ Myrinet Cluster } \\
\hline \# proc & $\begin{array}{c}\text { Processor } \\
\text { distribution }\end{array}$ & $\begin{array}{c}\text { LU-decomposition } \\
\text { (sec) }\end{array}$ & $\begin{array}{c}\text { LU-decomposition } \\
\text { (Flops) }\end{array}$ & $\begin{array}{c}\text { LU-decomposition } \\
\text { (sec) }\end{array}$ & $\begin{array}{l}\text { LU-decomposition } \\
\text { (Flops) }\end{array}$ \\
\hline 1 & $1 \times 1$ & 0.14 & 56.14 & 0.11 & 57.14 \\
\hline 2 & $1 \times 2$ & 10.14 & 5.19 & 0.24 & 25.49 \\
\hline 2 & $2 \times 1$ & 12.29 & 2.19 & 0.13 & 48.16 \\
\hline 4 & $1 \mathrm{x} 4$ & 13.11 & 0.42 & 1.90 & 3.17 \\
\hline 4 & $2 \times 2$ & 23.69 & 0.50 & 1.34 & 2.36 \\
\hline 4 & $4 \times 1$ & 11.13 & 0.42 & 0.41 & 14.57 \\
\hline 8 & $1 \times 8$ & - & - & 3.36 & 1.80 \\
\hline 8 & $2 \times 4$ & - & - & 2.56 & 2.36 \\
\hline 8 & $4 \times 2$ & - & - & 1.39 & 4.33 \\
\hline 8 & $8 \times 1$ & - & - & 0.34 & 17.62 \\
\hline
\end{tabular}

Table 3. Performance of the Myrinet cluster on the parallel solution of $A x=b$, with $\operatorname{dim}(A)=7 \times 10^{5}$ elements

\begin{tabular}{cccccc}
\multicolumn{5}{c}{ Myrinet Cluster } \\
\hline \# proc & $\begin{array}{c}\text { Processor } \\
\text { distribution }\end{array}$ & $\begin{array}{c}\text { LU-decomposition } \\
\text { (sec) }\end{array}$ & $\begin{array}{c}\text { Solve Time LU-decomposition } \\
\text { (sec) }\end{array}$ & $\begin{array}{c}\text { Solve } \\
\text { (Flops) }\end{array}$ \\
\hline 1 & $1 \times 1$ & 4.15 & 0.25 & 1159.12 & 86.56 \\
2 & $1 \times 2$ & 2.65 & 0.24 & 1815.61 & 88.70 \\
2 & $2 \times 1$ & 2.43 & 0.18 & 1981.04 & 117.86 \\
4 & $1 \times 4$ & 1.96 & 0.55 & 2451.85 & 39.13 \\
4 & $2 \times 2$ & 1.87 & 0.17 & 2570.00 & 127.75 \\
4 & $4 \times 1$ & 2.20 & 0.19 & 2184.66 & 113.46 \\
6 & $1 \times 6$ & 1.89 & 0.63 & 2548.34 & 34.57 \\
6 & $2 \times 3$ & 1.85 & 0.35 & 2599.90 & 6.80 \\
6 & $3 \times 2$ & 1.92 & 0.16 & 2506.08 & 13.17 \\
6 & $6 \times 1$ & 2.62 & 0.20 & 1834.86 & 110.03 \\
8 & $1 \times 8$ & 1.98 & 0.83 & 2434.88 & 25.98 \\
8 & $8 \times 1$ & 2.80 & 0.20 & 1722.45 & 108.89 \\
\hline
\end{tabular}




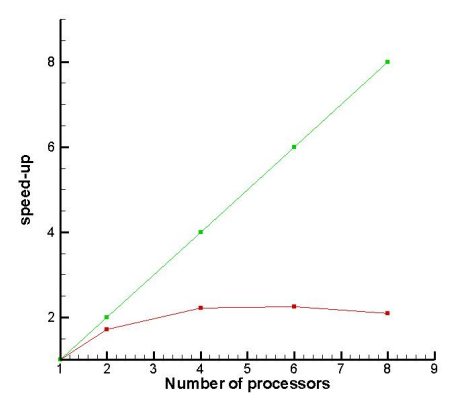

Fig. 3. Speed-up for the LU-decomposition in several processors. Theoretical (achieved in the case of two processors) result is shown in green; results of table 3 shown in red.

\section{References}

1. Theofilis, V., "Advances in global linear instability analysis of nonparallel and three-dimensional flows," Prog. Aero. Sci., Vol. 39, 2003, pp. 249-315.

2. Li, X. S. and Demmel, J. W., "SuperLUDIST: A Scalable Distributed-Memory Sparse Direct Solver for Unsymmetric Linear Systems," ACM Trans. Mathematical Software, Vol. 29, No. 2, June 2003, pp. 110-140.

3. de Vicente, J., Valero, E., Gonzalez, L., and Theofilis, V., "Spectral multidomain methods for BiGlobal instability analysis of complex flows over open cavity configurations," , No. AIAA Paper 2006-2877, 2006. 